\title{
Secreted Frizzled-related protein 4 inhibits the regeneration of hair follicles
}

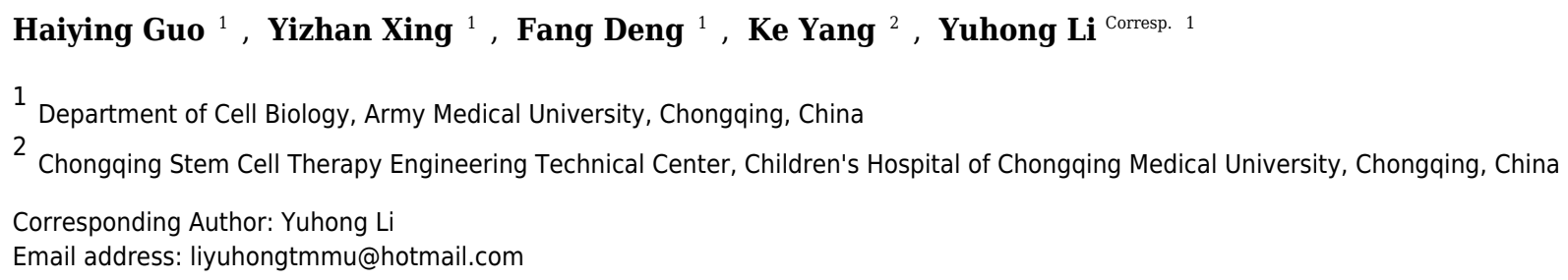

Secreted Frizzled-related Protein 4 (SFRP4) belongs to Wnt inhibitors. Previously, we reported that SFRP4 inhibited the differentiation of melanocyte. Here, by using of immunostaining, we showed that SFRP4 is expressed in both human and mouse hair follicles, especially in the outer root sheath and inner root sheath. To reveal the role of sFRP4 in hair follicle growth and hair cycle, we induced synchronized hair cycle in the dorsal skin of mice by depilation, and injected sFRP4 intradermally into the skin. By H\&E staining, we found that the regeneration of hair follicles was inhibited by SFRP4. However, the structure of hair follicles remained complete. Compared with PBS-treated hair follicles, the sFRP4-treated hair follicles still had the same expression pattern of keratins. Our findings reveal that SFRP4 inhibits but not blocks the regeneration of hair follicles, and supply a potential therapeutic application to treat hair follicle regeneration disorders. 
1

2

3

\section{Address:}

51 Department of Cell Biology, Army Medical University, Gaotanyan Street No. 30, Shapingba District,

6 Chongqing 400038, China.

72 Chongqing Stem Cell Therapy Engineering Technical Center, Children's Hospital of Chongqing Medical

8 University, Chongqing 400014, China.

$9 \quad$ *Corresponding author

Yuhong Li: Tel: +86 23 68771372, E-mail: liyuhongtmmu@,hotmail.com

11

\section{Secreted Frizzled-related protein 4 inhibits the regeneration of hair follicles}

Haiying Guo ${ }^{1}$, Yizhan Xing ${ }^{1}$, Fang Deng ${ }^{1}$, Ke Yang ${ }^{2}$, Yuhong Li ${ }^{1}{ }^{1 *}$

\begin{abstract}
Secreted Frizzled-related Protein 4 (sFRP4) belongs to Wnt inhibitors. Previously, we reported that sFRP4 inhibited the differentiation of melanocyte. Here, by using of immunostaining, we showed that sFRP4 is expressed in both human and mouse hair follicles, especially in the outer root sheath and inner root sheath. To reveal the role of sFRP4 in hair follicle growth and hair cycle, we induced synchronized hair cycle in the dorsal skin of mice by depilation, and injected sFRP4 intradermally into the skin. By H\&E staining, we found that the regeneration of hair follicles was inhibited by sFRP4. However, the structure of hair follicles remained complete. Compared with PBS-treated hair follicles, the sFRP4-treated hair follicles still had the same expression pattern of keratins. Our findings reveal that SFRP4 inhibits but not blocks the regeneration of hair follicles, and supply a potential therapeutic application to treat hair follicle regeneration disorders.
\end{abstract}




\section{Introduction}

The Wnt proteins play essential roles in the development, proliferation, differentiation and migration of various cell types. Wnts transduce signals by binding to Frizzled (Fz) receptor family and low-density lipoprotein-related protein 5/6 (LRP5/6) co-receptors. Activation of Wnt signaling is also controlled by the extracellular antagonists that can be divided into two classes, the secreted Frizzled-related proteins (sFRP) class and the Dickkopf (DKK) class. The sFRP class includes sFRP1, sFRP2, sFRP3, sFRP4, sFRP5, Wnt inhibitory factor 1(WIF1) and Cerberus. They antagonize Wnt signaling by interacting with Wnt proteins directly. The DKK class includes DKK1, DKK2, DKK3, DKK4. They antagonize Wnt signaling by binding to LRP5/6 co-receptor complex.

SFRP4 is a member of the sFRP family and has been reported to play roles in cell proliferation, differentiation, apoptosis and carcinogenesis (Drake et al. 2009; Ford et al. 2013; Huang et al. 2010; Maganga et al. 2008; Park et al. 2008). Recently, several studies have demonstrated the importance of sFRP4 in skin tissue (Bai et al. 2015; Bayle et al. 2008; Chen et al. 2014; Maganga et al. 2008). In lesional skin of psoriasis patients and mouse models, the expression of sFRP4 were significantly decreased at both mRNA level and protein level. On the contrast, the expression level of sFRP4 protein was increased in the skin of the systemic sclerosis patients and tight-skin mouse compared to healthy skin (Bai et al. 2015; Bayle et al. 2008). In cultured human keratinocytes, exogenous sFRP4 inhibited the proliferation of keratinocyte (Bai et al. 2015; Maganga et al. 2008). Furthermore, sFRP4 also promotes the apoptosis and differentiation of human keratinocytes in vitro (Maganga et al. 2008). Previous studies also reported that sFRP4 functioned as an extra-follicular modulator, and coordinated the hair follicle cycling behavior (Chen \& Chuong 2012; Chen et al. 2014; Plikus et al. 2011).

Previously, we have investigated the role of sFRP4 in melanogenesis. We observed that sFRP4 was preferentially expressed in keratinocytes adjacent to melanocytes in epidermis. However, the role of sFRP4 in hair follicle regeneration has not been well elucidated. To address this issue, we determined the expression pattern of sFRP4 in the skin of human and mouse, treated the mouse skin with sFRP4 protein, and observed the treated skin and hair follicles systematically. We found that sFRP4 inhibited the regeneration of hair follicles.

\section{Materials and Methods}

\subsection{Animals and skin samples}

C57 BL/6 mice were obtained from and housed in the laboratory animal center of the Army Medical University. All the animal-related procedures were in strict accordance with the approved institutional animal care and maintenance protocols. All experimental protocols were approved by the Laboratory Animal Welfare and Ethics Committee of the Third Military Medical University. Permission number for producing animals: SCXK (YU)-20170002. Permission number for using animals: SYXK (YU)-20170002.

Human skin samples were obtained from 30 to 40 years old donors in the second affiliated Hospital of the Army Medical University. The use of the samples was consented verbally by the donors. The research was approved by the ethics committee of the Third Military Medical University.

\subsection{Immunofluorescence}

Immunostaining was performed on $5 \mu \mathrm{m}$ sections from tissue samples. Sections were dewaxed, rehydrated, and boiled in citrate buffer solution. Then the sections were blocked with $10 \%$ goat serum, incubated with the 
following primary antibodies: goat anti-sFRP4 (1:100, Abcam, China), rabbit anti-K6 (1:50, Sangon Biotech, China), rabbit anti-K10 (1:50, Sangon Biotech, China), rabbit anti-K14 (1:50, Sangon Biotech, China), and rabbit anti-K19 (1:50, Sangon Biotech, China), and rabbit anti- $\beta$-catenin (1:200, Abcam, USA). After washing, the sections were incubated with Alexa Fluor 488 (Invitrogen, USA) or Cy3 (Beyotime, China) labeled secondary antibodies. After that, sections were counterstained with DAPI for nuclei visualization. At last, the cover slides were moved to microscope slides, mounted with antifade mounting medium (Beyotime, China), and observed under fluorescent microscope.

\subsection{Intradermal administration}

To induce synchronized hair cycle, the hairs on the dorsal skin of 7-week-old C57 mice were depilated as described previously (Muller-Rover et al. 2001). Twenty-five $\mu \mathrm{L}$ recombinant sFRP4 (50 $\mu \mathrm{g} / \mathrm{mL}$, R\&D Systems, USA) or PBS (control) was administered intradermally into the dorsal skin after depilation. At 2 days, 3 days, or 4 days after the administration of sFRP4, the dorsal skin samples were harvested and prepared for analysis of histology or immunostaining.

\subsection{Hematoxylin and eosin (H\&E) staining}

The skin collected from sFRP4 or PBS administrated region were fixed with 4\% paraformaldehyde overnight, gradually dehydrated in graded alcohol solutions, cleared in xylene, and subsequently embedded in paraffin. Sectioned samples were dewaxed, rehydrated, stained with hematoxylin (ZSGB-bio, China) for 2 minutes and subsequently rinsed with water. The sections were later stained with eosin (ZSGB-bio, China) for 2 minutes and rinsed with water thereafter. At last, the sections were dehydrated gradually, mounted with neutral gum (ZSGBbio, China) and observed under a microscope.

\subsection{Statistical analysis}

Data were presented as a mean $\pm \mathrm{SD}$ of three independent experiments. Statistical analyses were evaluated using t-test and $\mathrm{p}<0.05$ was considered statistically significant.

\section{Results}

\section{1 sFRP4 is expressed in human and mouse hair follicles}

At first, we detected the endogenous expression of sFRP4 in human scalp skin by immunofluorescence. In epidermis, sFRP4 was expressed in all the layers (Fig. 1A-1C). In the human hair follicle, sFRP4 was strongly expressed in the inner root sheath (IRS) and the outer root sheath (ORS), weaker in the hair shaft (HS) and precortex (Fig. 1D-1I).

Then we detected the expression pattern of sFRP4 in mouse dorsal skin. Among all the hair cycle stages, sFRP4 was widely expressed in the hair follicle (Fig. 2A-2I). In anagen, nearly all the hair follicle structures can be observed. Double-labeling immunostaining further confirmed that sFRP4 was expressed at the ORS (K14 positive) and IRS (K10 positive) region in anagen hair follicles. The expression of sFRP4 was also detected in the IRS precursors and hair matrix region of mouse hair follicles (Fig. 2J-2Q).

\section{2 sFRP4 inhibits the growth of hair follicle in vivo}


101

102

103

104

105

106

107

108

109

110

111

112

113

114

115

116

117

118

119

120

121

122

123

124

125

126

127

128

129

130

131

132

133

134

135

136

The expression pattern of sFRP4 in hair follicle suggests that sFRP4 may play some roles in hair follicle cycle. To find out the roles of sFRP4 in hair follicle cycle and hair follicle regeneration, we induced synchronized hair growth by depilation and injected sFRP4 protein intradermally into the mouse dorsal skin (Fig. 3A). At 2 days after injection, the regeneration of the hair follicle was inhibited. However, the regeneration of the hair follicle was not blocked, it went on growing after the injection of sFRP4 stopped (Fig. 3C-3J). We also measured the length of hair follicles in the pictures of H\&E staining with Image Pro Plus 6.0 software. Statistical analysis revealed that hair follicles in the sFRP4-injected group showed decreased hair length at 4 days after depilation (Fig. 3B).

\section{3 sFRP4 does not affect the expression pattern of structure markers in hair follicles}

K6, K10, K14, and K19 were expressed in the regenerated hair follicles, both in the PBS-treated group and sFRP4-treated group. K6 and K14 were expressed in both IRS and ORS. K10 was expressed in the IRS.K19 was expressed in the bulge region (Fig. 4). The expression patterns were similar between the two groups, but the regeneration of hair follicles in sFRP4-treated group was delayed.

\section{4 sFRP4 inhibits the nuclear translocation of $\beta$-catenin in hair follicles}

Beta-catenin expressed in the regenerated hair follicles, both in the PBS-treated group and sFRP4-treated group. In PBS-treated group, $\beta$-catenin was expressed in both nucleus and cell plasma (Fig. 5A, 5C). At 2 days after treatment, only few nuclear translocation of $\beta$-catenin was observed (Fig. 5A). At 4 days after treatment, more nuclear translocation of $\beta$-catenin was observed (Fig. 5C). In sFRP4-treated group, $\beta$-catenin was expressed in cell plasma (Fig. 5B, 5D).

\section{Discussion}

Hair follicle grows periodically. The hair cycle is consist of anagen, catagen, and telogen. The regeneration of hair follicles begins with anagen. It is regulated by many factors, including factors from macroenvironment such as the level of hormone and mood, and factors from microenvironment such as adjacent cells. Secreted factors impact the regeneration of hair follicles through autocrine, paracrine, or endocrine. sFRP4 is a member of secreted Frizzled-related proteins. It is reported that sFRP4 took effects via autocrine or paracrine (Bafico et al. 1999). sFRP4 is expressed in various tissues normally including endometrial stroma, pancreas, stomach, colon, lung, skeletal muscle, testis, ovary, kidney, heart, brain, mammary gland, cervix, eye, bone, prostate, and liver (Pawar \& Rao 2018). In the hair follicle, we found that sFRP4 was expressed in nearly all the keratinocytes, including ORS, IRS, matrix and epidermis. As to hair cycle, sFRP4 was expressed in all the stages, including anagen, catagen, and telogen, which is in accordance with previous report (Chen et al. 2014). This expression pattern suggests that sFRP4 may play some roles in all the layers of hair follicle and epidermis. The expression pattern of sFRP4 in human scalp was similar to the expression pattern of sFRP4 in mouse dorsal skin. This suggests that sFRP4 may play similar role in different species.

After depilation, hair follicles enter anagen immediately (Muller-Rover et al. 2001). When sFRP4 was injected into the mouse skin, the regeneration of hair follicle was inhibited. The structures of ORS, IRS and matrix were smaller than the control, and the length of hair follicle was shorter. However, the hair follicle 
entered anagen as well. This suggests that sFRP4 may not block the anagen onset, but inhibit the structure formation of hair follicle. Previously, we reported that sFRP4 inhibited the differentiation of melanocytes (Guo et al. 2017). Here, sFRP4 may play its role via inhibiting the proliferation and differentiation of precortex cells, transit amplifying cells, and hair follicle stem cells.

Usually, sFRPs take effects by binding with Wnt ligands, thus blocking the interaction of Wnt ligands and Wnt receptors. However, sFRPs can also augment Wnt signaling under certain conditions (Kawano \& Kypta 2003). Canonical Wnt ligands such as Wnt3a and Wnt10b can induce hair follicle regeneration (Li et al. 2013). Non-canonical Wnt ligands such as Wnt5a could inhibit the anagen onset of hair cycle (Xing et al. 2016). Translocation of $\beta$-catenin from plasma to nucleus is a key point for Wnt signaling pathway. Blocking of the translocation of $\beta$-catenin inhibited the anagen onset of hair regeneration. Here, sFRP4 inhibited the growth of hair follicle. It also inhibited the nuclear translocation of $\beta$-catenin. It is similar to the situation of the inhibition of canonical Wnt signaling pathway. Thus, sFRP4 may take effects by binding with Wnt ligands.

\section{Conclusions}

From the results of the present study, we conclude that sFRP4 is expressed in both mouse and human hair follicles. During anagen, overexpression of sFRP4 inhibits the growth of hair follicles through inhibiting the nuclear translocation of $\beta$-catenin, but would not block the growth of hair follicles. The hair follicles regenerated from sFRP4 treated skin have complete hair follicle structure.

\section{Acknowledgements}

We thank Prof. Jin Yang for her constructive suggestions during the experiments.

\section{References}

Bafico A, Gazit A, Pramila T, Finch PW, Yaniv A, and Aaronson SA. 1999. Interaction of frizzled related protein (FRP) with Wnt ligands and the frizzled receptor suggests alternative mechanisms for FRP inhibition of Wnt signaling. J Biol Chem 274:16180-16187.

Bai J, Liu Z, Xu Z, Ke F, Zhang L, Zhu H, Lou F, Wang H, Fei Y, Shi YL, and Wang H. 2015. Epigenetic downregulation of SFRP4 contributes to epidermal hyperplasia in psoriasis. J Immunol 194:4185-4198.

Bayle J, Fitch J, Jacobsen K, Kumar R, Lafyatis R, and Lemaire R. 2008. Increased expression of Wnt2 and SFRP4 in Tsk mouse skin: role of Wnt signaling in altered dermal fibrillin deposition and systemic sclerosis. J Invest Dermatol 128:871-881.

Chen CC, and Chuong CM. 2012. Multi-layered environmental regulation on the homeostasis of stem cells: the saga of hair growth and alopecia. $J$ Dermatol Sci 66:3-11.

Chen CC, Murray PJ, Jiang TX, Plikus MV, Chang YT, Lee OK, Widelitz RB, and 
173

174

175

176

177

178

179

180

181

182

183

184

185

186

187

188

189

190

191

192

193

194

195

196

197

198

199

200

201

202

203

204

205

206

207

208

209

210

211

212

213

Chuong CM. 2014. Regenerative hair waves in aging mice and extrafollicular modulators follistatin, dkk1, and sfrp4. J Invest Dermatol 134:2086-2096.

Drake J, Shearwood AM, White J, Friis R, Zeps N, Charles A, and Dharmarajan A. 2009. Expression of secreted frizzled-related protein 4 (SFRP4) in primary serous ovarian tumours. Eur J Gynaecol Oncol 30:133-141.

Ford CE, Jary E, Ma SS, Nixdorf S, Heinzelmann-Schwarz VA, and Ward RL. 2013. The Wnt gatekeeper SFRP4 modulates EMT, cell migration and downstream Wnt signalling in serous ovarian cancer cells. PLoS One 8:e54362.

Guo H, Lei M, Li Y, Liu Y, Tang Y, Xing Y, Deng F, and Yang K. 2017. Paracrine Secreted Frizzled-Related Protein 4 Inhibits Melanocytes Differentiation in Hair Follicle. Stem Cells Int 2017:2857478.

Huang D, Yu B, Deng Y, Sheng W, Peng Z, Qin W, and Du X. 2010. SFRP4 was overexpressed in colorectal carcinoma. J Cancer Res Clin Oncol 136:395401.

Kawano Y, and Kypta R. 2003. Secreted antagonists of the Wnt signalling pathway. J Cell Sci 116:2627-2634.

Li YH, Zhang K, Yang K, Ye JX, Xing YZ, Guo HY, Deng F, Lian XH, and Yang T. 2013. Adenovirus-mediated Wnt10b overexpression induces hair follicle regeneration. J Invest Dermatol 133:42-48.

Maganga R, Giles N, Adcroft K, Unni A, Keeney D, Wood F, Fear M, and Dharmarajan A. 2008. Secreted Frizzled related protein-4 (sFRP4) promotes epidermal differentiation and apoptosis. Biochem Biophys Res Commun 377:606-611.

Muller-Rover S, Handjiski B, van der Veen C, Eichmuller S, Foitzik K, McKay IA, Stenn KS, and Paus R. 2001. A comprehensive guide for the accurate classification of murine hair follicles in distinct hair cycle stages. $J$ Invest Dermatol 117:3-15.

Park JR, Jung JW, Lee YS, and Kang KS. 2008. The roles of Wnt antagonists Dkk1 and sFRP4 during adipogenesis of human adipose tissue-derived mesenchymal stem cells. Cell Prolif 41:859-874.

Pawar NM, and Rao P. 2018. Secreted frizzled related protein 4 (sFRP4) update: A brief review. Cell Signal 45:63-70.

Plikus MV, Baker RE, Chen CC, Fare C, de la Cruz D, Andl T, Maini PK, Millar SE, Widelitz R, and Chuong CM. 2011. Self-organizing and stochastic behaviors during the regeneration of hair stem cells. Science 332:586589.

Xing Y, Ma X, Guo H, Deng F, Yang J, and Li Y. 2016. Wnt5a Suppresses betacatenin Signaling during Hair Follicle Regeneration. Int J Med Sci $13: 603-610$. 


\section{Figure 1}

The expression of sFRP4 in human scalp

(A-C) The expression of sFRP4 in epidermis. (D-F) The expression of sFRP4 in the bulge area of hair follicles. (G-I) The expression of sFRP4 in the hair bulb area of hair follicles. The nuclei were counterstained with DAPI. The right panel $(C, F, I)$ is the merge of left two panels $(A, B$, D, E, G, H). Ep, epidermis. HS, hair stem. ORS, outer root sheath. IRS, inner root sheath. Co, precortex. DP, dermal papilla. Scale bar $=50 \mu \mathrm{m}$.

*Note: Auto Gamma Correction was used for the image. This only affects the reviewing manuscript. See original source image if needed for review.

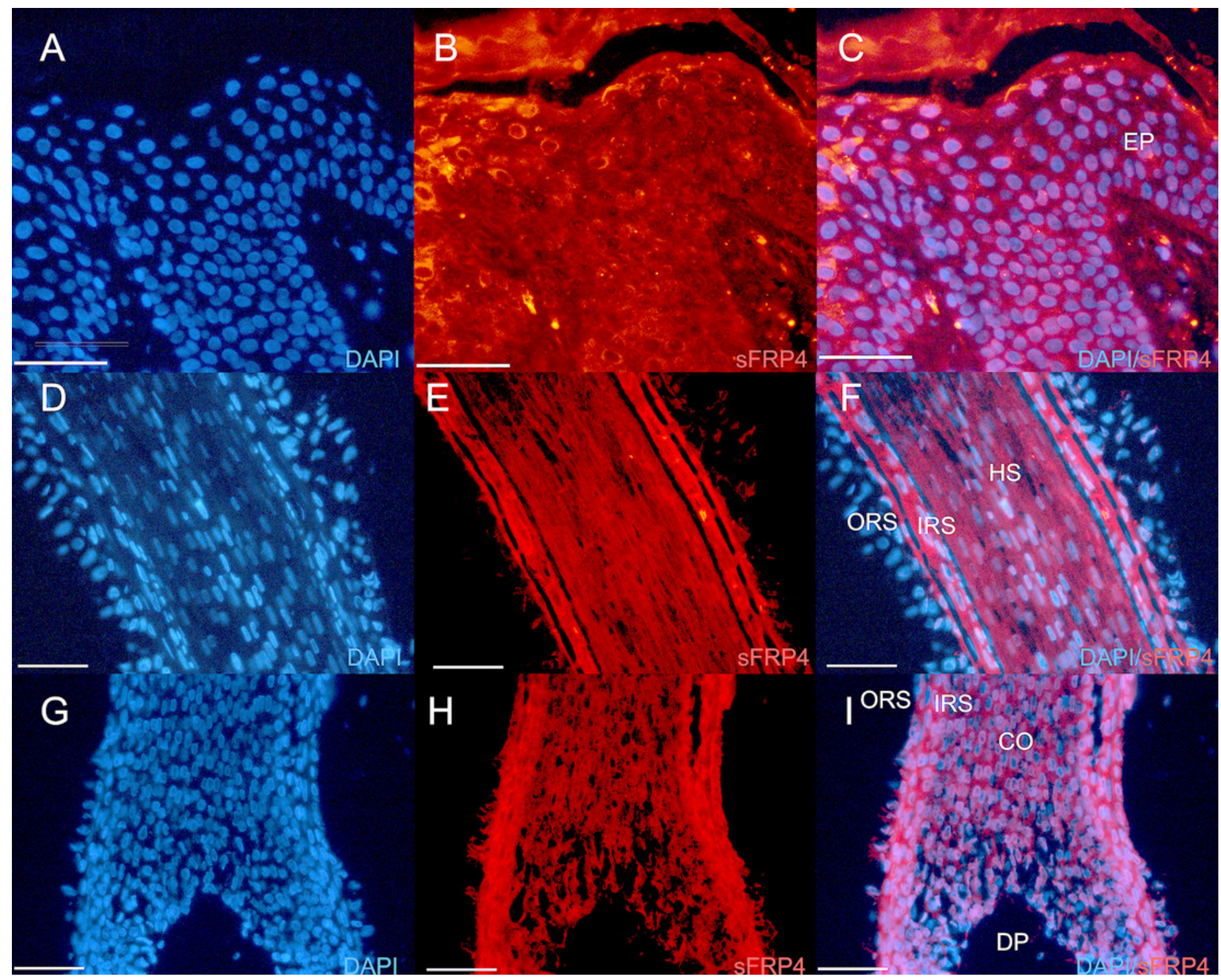




\section{Figure 2}

The expression of sFRP4 in mouse dorsal skin

(A-I) The expression of sFRP4 (red color) in hair cycle. (A-C) Postnatal day 8, anagen. (D-F) Postnatal day 16, catagen. (G-I) Postnatal day 21, telogen. (J-M) The expression of sFRP4 (red color) and K14 (green color) in anagen mouse skin. (N-Q) The expression of sFRP4 (red color) and $\mathrm{K} 10$ (green color) in anagen mouse skin. The right panel $(\mathrm{C}, \mathrm{F}, \mathrm{I}, \mathrm{M}, \mathrm{Q})$ is the merge of left panels $(A, B, D, E, G, H, J-L, N-P)$. The nuclei were counterstained with DAPI. Scale bar = $50 \mu \mathrm{m}$.

*Note: Auto Gamma Correction was used for the image. This only affects the reviewing manuscript. See original source image if needed for review. 


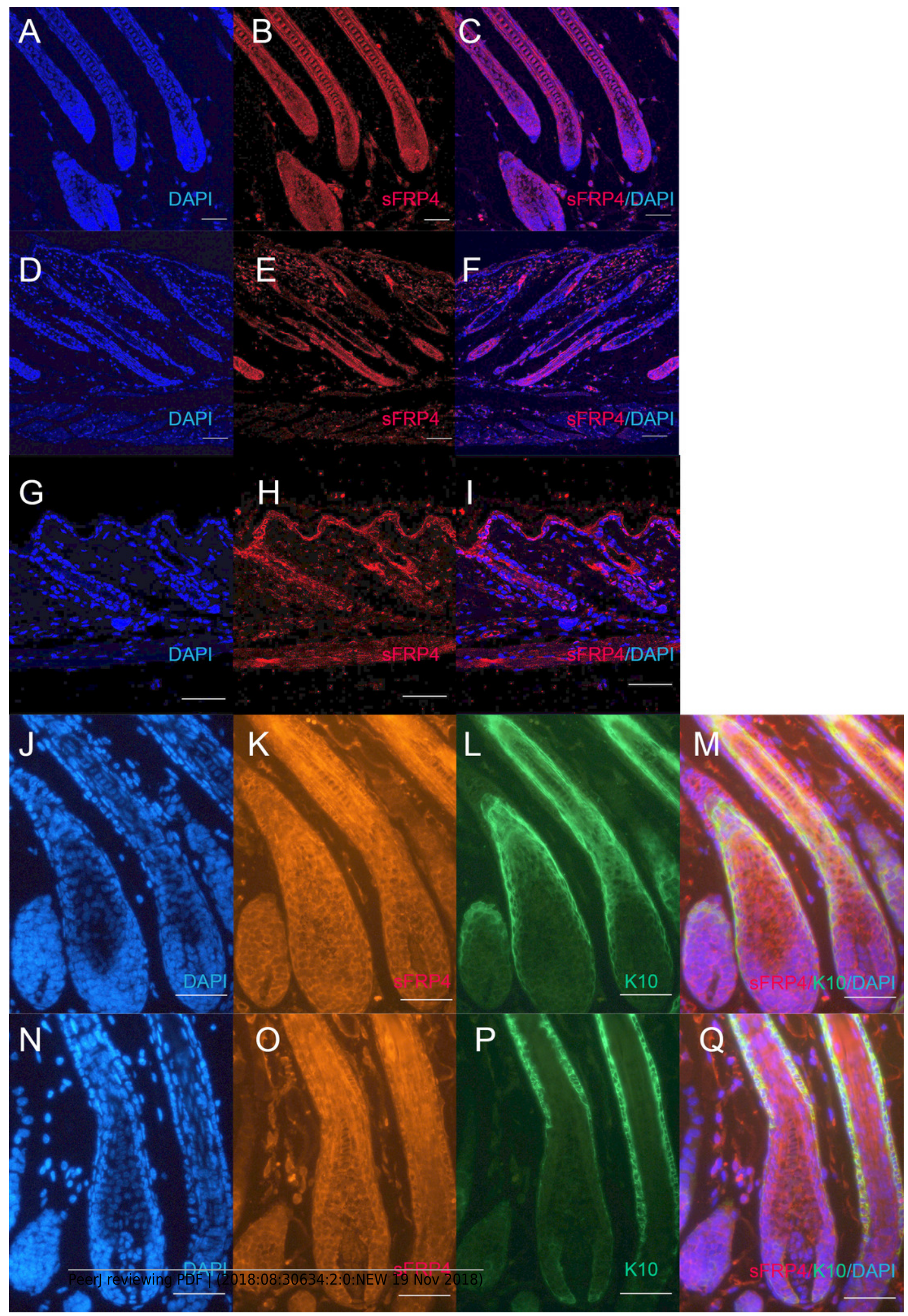




\section{Figure 3}

SFRP4 inhibited the growth of hair follicle

(A) The working model for the animal experiment. The hairs of 7-week-old mice were depilated, and SFRP4 was injected. The samples were obtained at day 2 and day 4 post injection. (B) The length of regenerated hair follicles in PBS-treated or SFRP4-treated mouse skin. $\mathrm{N}=3, * \mathrm{P}<0.05$. (C-J) H\&E staining displays the structure of hair follicles in the PBStreated or sFRP4-treated mouse skin. The right panel $(D, F, H, J)$ is the enlargement of the framed area in the left panel (C, E, G, I). 2d-PBS, 4d-PBS, 2 days or 4 days after the injection of PBS. 2d-sFRP4, 4d-sFRP4, 2 days or 4 days after the injection of sFRP4. Scale bar $=100$ $\mu \mathrm{m}$. 

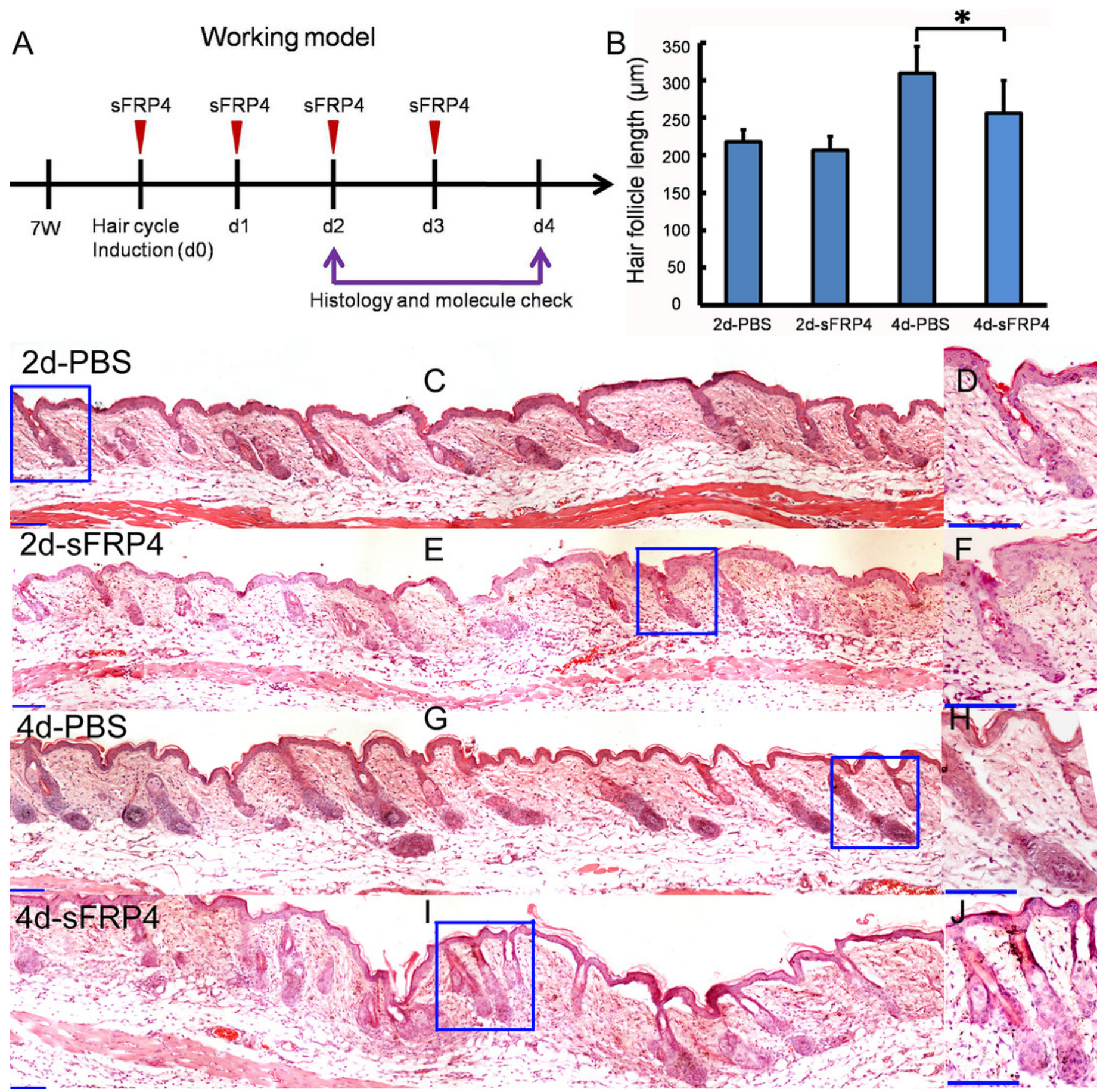


\section{Figure 4}

The expression of keratins in regenerated hair follicle

The expression of keratins $K 6(A, E), K 10(B, F), K 14(C, G), K 19(D, H)$ were determined by immunoflurescence. (A-D) The expression of keratins in sFRP4-injected samples. (E-H) The expression of keratins in PBS-injected samples. The nuclei were counterstained with DAPI.

Scale bar $=50 \mu \mathrm{m}$.

*Note: Auto Gamma Correction was used for the image. This only affects the reviewing manuscript. See original source image if needed for review.

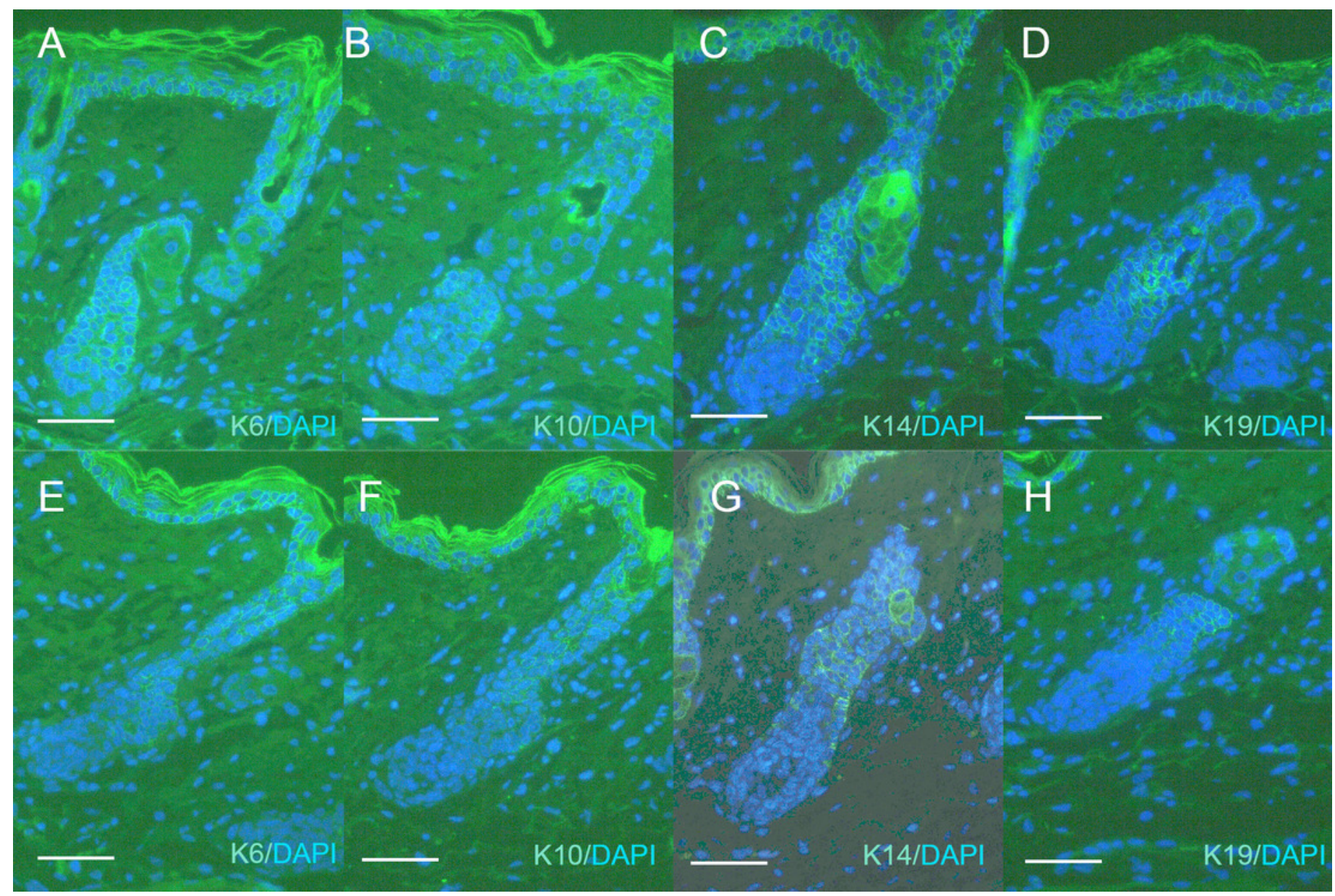




\section{Figure 5}

The expression of $\beta$-catenin in regenerated hair follicle

The expression of $\beta$-catenin was determined by immunofluorescence. (A-B) The expression of $\beta$-catenin at 2 days after treatment. (C-D) The expression of $\beta$-catenin at 4 days after treatment. (A) and (C) show the expression of $\beta$-catenin in PBS-injected samples. (B) and (D) show the expression of $\beta$-catenin in sFRP4-injected samples. The nuclei were counterstained with DAPI. Scale bar $=10 \mu \mathrm{m}$.

*Note: Auto Gamma Correction was used for the image. This only affects the reviewing manuscript. See original source image if needed for review. 


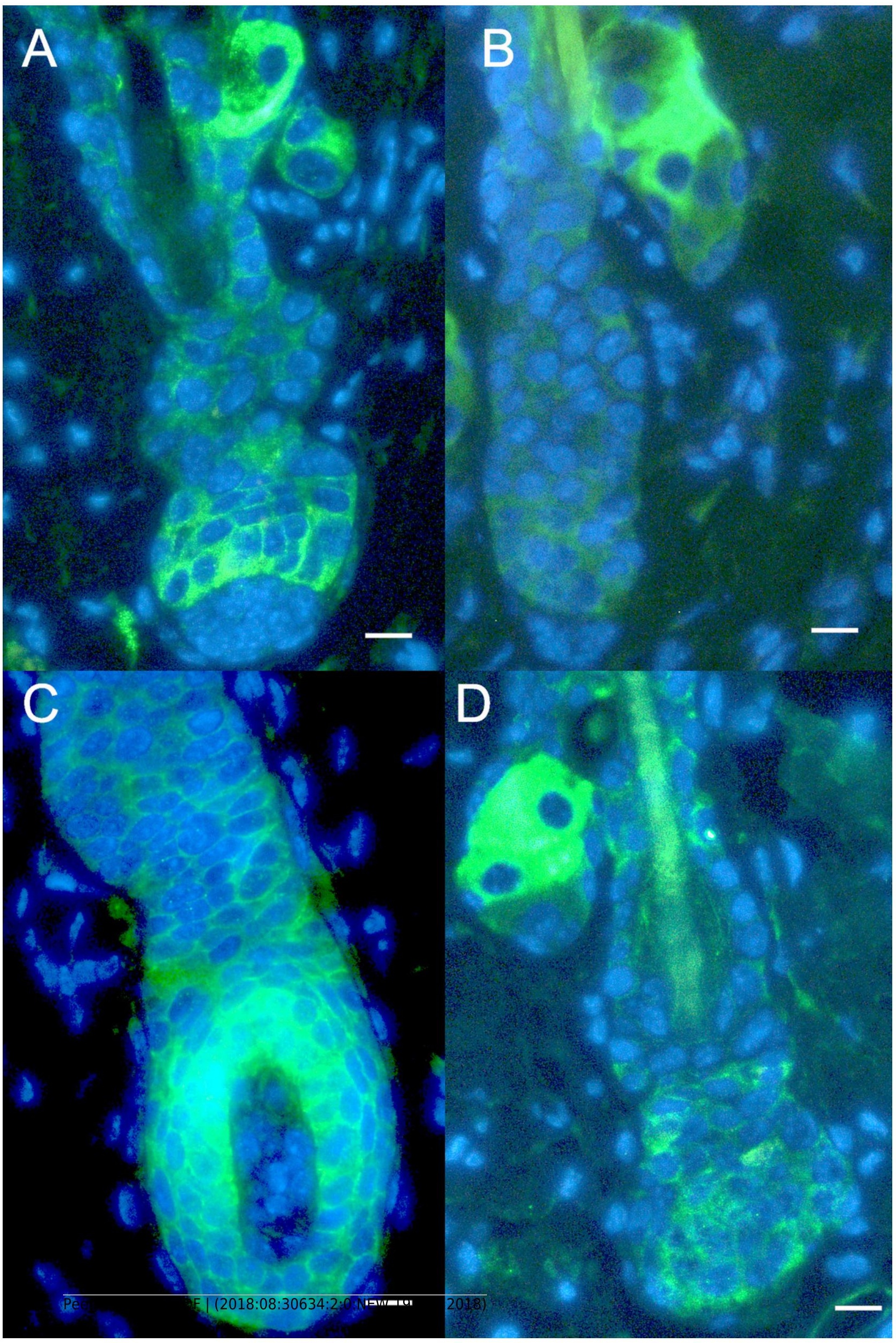

